http://jmscr.igmpublication.org/home/

ISSN (e)-2347-176x ISSN (p) 2455-0450

crossref DOI: https://dx.doi.org/10.18535/jmscr/v7i8.38

Journal Of Medical Science And Clinical Research

\title{
Radiological Evaluation of Hepatocellular Carcinoma
}

\author{
Authors \\ Dr L.V.V. Prasad MD (RD), Dr Sri Lakshmi J P Rao G, Dr Y. Trinadh \\ Department of Radio-Diagnosis, Andhra Medical College, Visakhapatnam, India
}

\begin{abstract}
Introduction: Hepatocellular carcinoma is the fifth most common cancer worldwide and the second leading cause of cancer-related death. More than 7 lakh people die of HCC each yearl,2. Imaging and histopathology plays a very important role in the diagnosis of HCC.

Materials \& Methods: This $s$ an observational study conducted over a period of one year, from September 2016 to October 2017 on 36 patients aged between 20-80yrs with sonologically detected hepatic mass lesions suspicious for HCC. They were evaluated with 16 slice CECT and 1.5T MRI. After taking informed consent, USG guided Biopsy/FNAC was done from these lesions and only those lesions that showed HCC were included in the study. The lesion characteristics are retrospectively analysed for the presence of specific features on US, CECT, MRI.

Results: Majority of the cases occurred in the age group of 51-60 years (38.88\%), and there is a male preponderance (83.33\%). Half the patients are cirrhotic (52.78\%). Hepatomegaly is observed in $55.56 \%$ cases. 50\% of the cases measured $5-10 \mathrm{~cm}$ in largest dimension. Majority of the lesions are hyperechoic and show intralesional vascularity. Majority of the lesions showed enhancement in the arterial phase of the CECT, followed by washout in the venous phase. Majority of them showed a well-defined capsule around the lesion, are hypointense on T1 and Hyperintense on T2, showing a mosaic architecture. Two thirds of the patients tested positive for Hepatitis B, deranged liver function tests and increased Alpha feto protein levels.

Conclusion: HCC can occur in a wide range of age groups. Though majority of the lesions show similar characteristics, HCC can have a wide spectrum of radiological appearances, and hence no single feature can be pathognomonic of the diagnosis. Hence, a tissue diagnosis is always desirable, whenever there is high index of suspicion, in lesions showing atypical pattern of imaging features.
\end{abstract}

\section{Introduction}

Hepatocellular carcinoma (HCC) is the fifth most common cancer worldwide and the second leading cause of cancer-related death. More than 7 lakh people die of HCC each year ${ }^{1,2}$ It occurs primarily in subjects who have chronic liver disease or liver cirrhosis $^{3}$ and is the primary cause of death among this group. The high-risk group includes patients testing positive for Hepatitis B, Hepatitis C, or those suffering with cirrhosis ${ }^{4}$. HCC usually occurs in the setting of cirrhosis3, although this is not always the case, with the classic example being HCC associated with hepatitis $B$ in the noncirrhotic liver. Several imaging diagnostic systems have been proposed for the interpretation of liver lesions in at-risk patients for HCC. Although these imaging-based diagnostic systems represent important advances, they have limitations and they are not perfectly consistent with each other ${ }^{5}$. Unfortunately, despite numerous 
technological developments and improvements in recent years, the diagnostic accuracy of noninvasive imaging in patients with cirrhosis is still relatively low, ranging respectively between 0.60 and 0.72 for USG, between 0.74 and 0.83 for MDCT and between 0.71 and 0.87 for $\mathrm{MRI}^{6-13}$. The relatively poor diagnostic performance for the detection of HCC in cirrhotic liver is due principally to overlapping imaging features and, thus, difficulties in differentiating dysplastic nodules from small HCC, and to problems associated with diagnosing arterially enhancing nodules smaller than $2 \mathrm{~cm}$ in diameter. For these reasons patients generally undergo regular followup with CT or MRI.

\section{Aims and Objectives}

1) To study the radiological behaviour of $\mathrm{HCC}$

2) To study the incidence of specific imaging features on Ultrasound, MDCT and MRI in pathologically proven cases of Hepatocellular carcinoma.

\section{Materials and Methods}

Study Design: Observational study

Study Period: One year from September 2016 to October 2017, at department of Radiodiagnosis, Andhra Medical College, Visakhapatnam.

\section{Inclusion Criteria}

The study group included patients with normal renal function (serum creatinine $<1.4 \mathrm{mg} / \mathrm{dl}$ ) with

1. Clinical suspicion of focal liver lesions.

2. Previous imaging studies depicted focal liver lesions with nonspecific appearance.

\section{Exclusion Criteria}

1. Patients with imaging features characteristic of benign hepatic lesion

2. Pregnancy

3. Acute or chronic renal failure

4. History of allergy to contrast media

5. Inability to tolerate sufficient breath hold.

6. Patients with pace makers or implants. Sample size: 36 cases.

\section{Results}

The youngest patient diagnosed with $\mathrm{HCC}$ in our study is 25 years and the oldest is 81 years. The average age of the patient at diagnosis is 54 years. Majority of the cases occurred in the age group of 51-60 years $(38.88 \%)$, followed by $61-70$ years $(25 \%)$ and $41-50$ years $(17.94 \%)$ in that order, of which 30 persons $(83.33 \%)$ are male and the rest female. Cirrhosis is noted in $19(52.78 \%)$ of the 36 patients, of which 2 are females and 17 are males. The youngest person with cirrhosis, diagnosed with HCC, is 40 years. Hepatomegaly is found in 20 people $(55.56 \%)$, more so with larger lesions. Of all the lesions studied, no lesion showed intra lesional calcification. Majority of the lesions studied measure between $5-10 \mathrm{~cm}$ in largest dimension, shown by 18 lesions, corresponding to $50 \%$. 8 lesions (22.22\%) measured $<5 \mathrm{~cm}$ and 7 lesions (19.44\%) measured $10-15 \mathrm{~cm}$. The average size of the lesion is $7.986 \mathrm{~cm}$ in largest dimension. Of the 36 lesions studied, 25 lesions, corresponding to $69.44 \%$ are hyperec-hoic, 9 lesions, corresponding to $25 \%$ are mixed echogenic and 2 lesions, corresponding to $5.56 \%$ are predominantly hypoechoic. Intralesional vascularity is seen in 30 persons, accounting for $83.33 \%$ and Peri-lesional halo could be demonstrated in 26 cases, corresponding to $72.22 \%$. Intralesional necrosis is seen in 15 cases $(41.67 \%) .7$ persons $(19.44 \%)$ have portal vein thrombosis. In this study, 35 lesions (97.22\%) showed enhancement in arterial phase and one lesion showed no enhancement (2.78\%). Venous phase washout was seen in 32 of the 35 lesions that showed enhancement in the arterial phase (91.43\%). While 3 lesions (8.57\%) did not show wash out in the venous phase. A welldefined delayed enhancing capsule could be demonstrated in 28 lesions (77.78\%).34 lesions $(94.44 \%)$ showed $\mathrm{T} 1$ isointense signal, one (2.77\%) showed T1 Hyperintense signal and one $(2.77 \%)$ remained isointense. 35 lesions (97.22\%) showed T2 Hyperintense signal while one lesion $(2.77 \%)$ showed hypo intensity on T2. 28 lesions (77.78\%) showed mosaic architecture while 8 $(22.22 \%)$ lesions did not. 
Table 1: Frequency of Cirrhosis

\begin{tabular}{|l|c|c|c|c|}
\hline & Male & Female & Total & \% \\
\hline Cirrhotic & 17 & 2 & 19 & $52.78 \%$ \\
\hline Non-Cirrhotic & 13 & 4 & 17 & $47.22 \%$ \\
\hline Total & 30 & 6 & 36 & $100 \%$ \\
\hline
\end{tabular}

Table 2: Pattern of Enhancement

\begin{tabular}{|l|c|c|}
\hline Pattern of Enhancement & Frequency & \% \\
\hline None & 1 & $2.78 \%$ \\
\hline Arterial & 35 & $97.22 \%$ \\
\hline Total & 36 & $100 \%$ \\
\hline
\end{tabular}

Table 3: Wash out Pattern

\begin{tabular}{|l|c|c|}
\hline Wash out pattern & Frequency & $\%$ \\
\hline Venous phase & 32 & $91.43 \%$ \\
\hline NA & 3 & $8.57 \%$ \\
\hline Total & 35 & $100 \%$ \\
\hline
\end{tabular}

Table 4: T1 \& T2 Signal Characteristics

\begin{tabular}{|l|c|c|}
\hline Signal & T1 & T2 \\
\hline Hypo intense & 34 & 1 \\
\hline Hyperintense & 1 & 35 \\
\hline Isointense & 1 & 0 \\
\hline Total & 36 & 36 \\
\hline
\end{tabular}

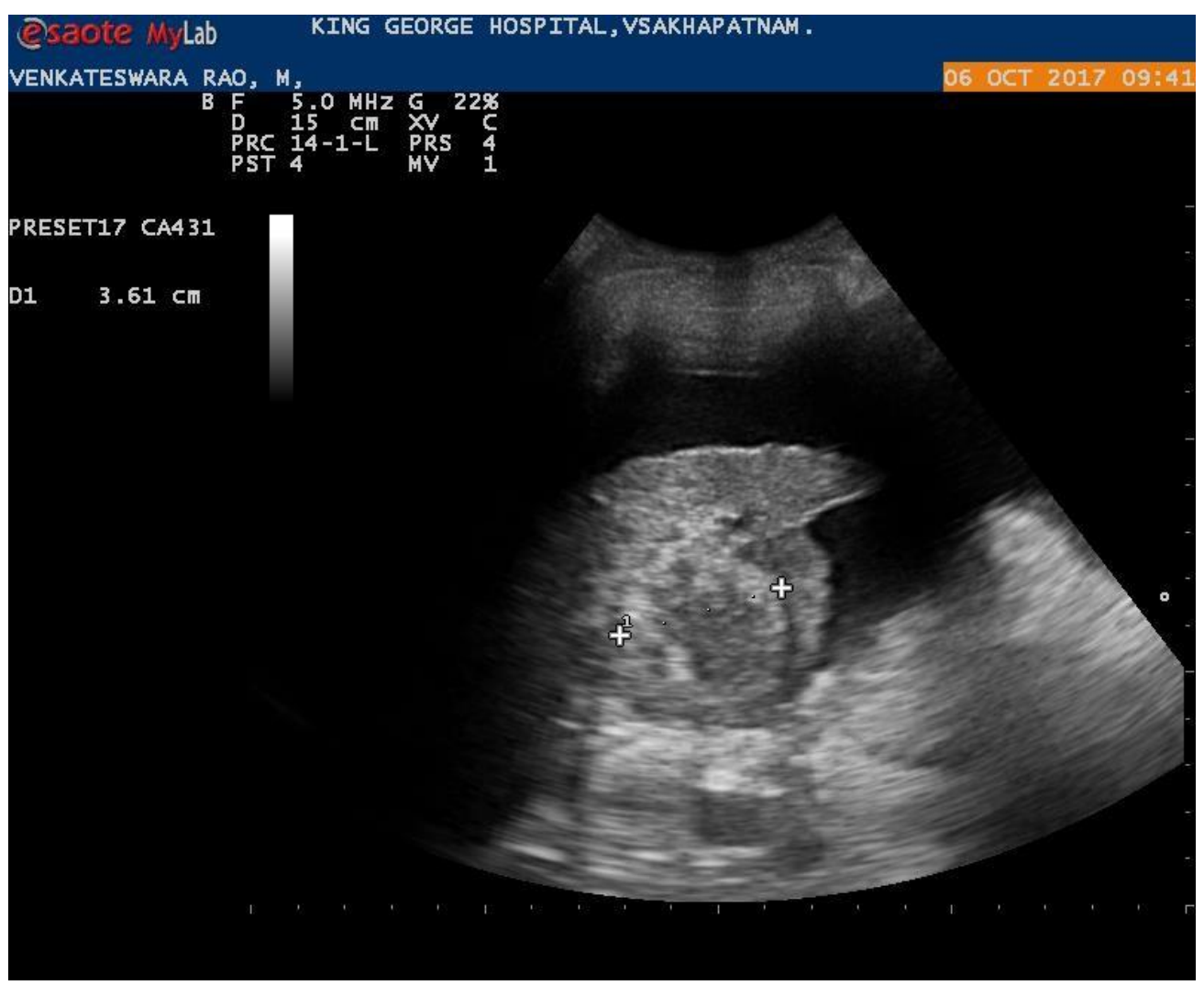

Figure 1: Ultrasound examination shows a well-defined hypoechoic perilesional halo in this 51-year-old male. 


\section{JMSCR Vol||07||Issue||08||Page 226-231||August}

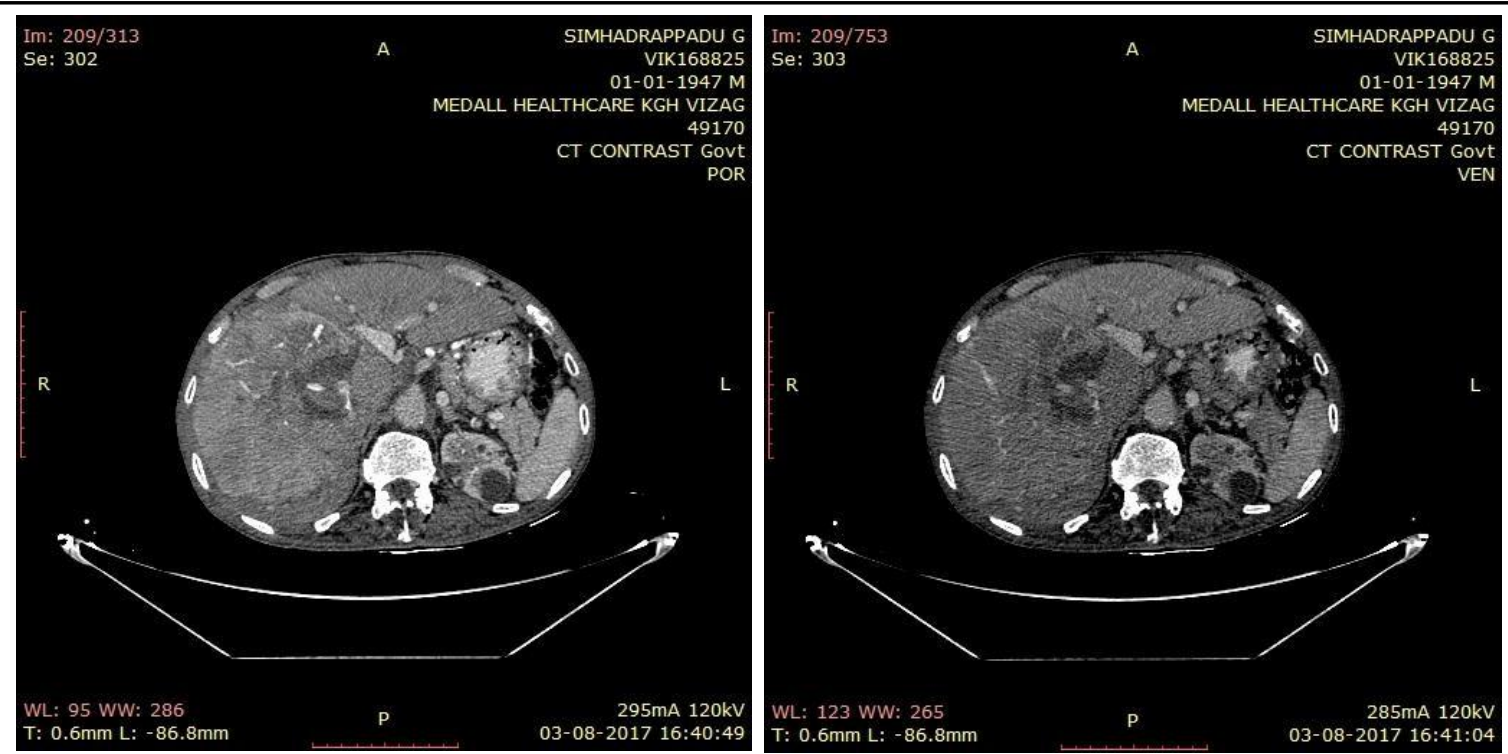

Figure 2: CECT in a 70-year-old man shows arterial phase enhancement of the lesion with subsequent washout in the venous phase.

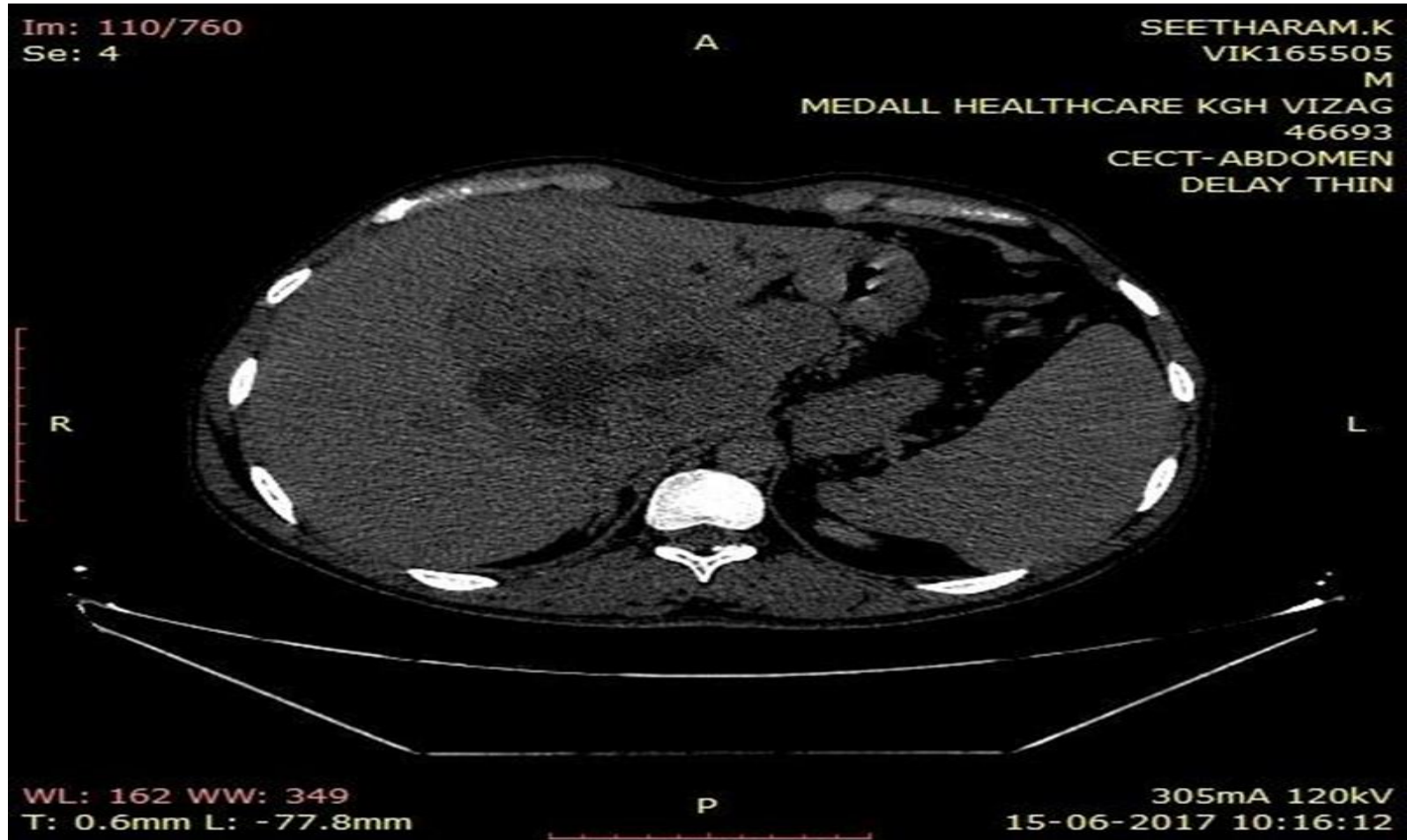

Figure 3: CECT in a 30-year-old male shows a well-defined capsule showing enhancement in the delayed phase.

\section{Discussion}

HCC can occur in a wide range of age groups including young patients. This is in contrast to the general opinion that HCC is a disease of the adults and elderly. The youngest patient in our study is 25 years old. The average age at diagnosis is 54 years. Cirrhosis is a known risk factor for the development of HCC, which is reflected in our study with $52.78 \%$ of the study group being cirrhotic. A male preponderance is noted with the disease, probably due to other risk factors like alcoholism, that predispose to Cirrhosis and eventually HCC. Hepatomegaly was observed with $55.56 \%$ of the cases, which doesn't correlate with the size of the tumour or with the no. of lesions. Majority of the lesions studied measure 
between $5-10 \mathrm{~cm}$ in largest dimension, shown by 18 lesions, corresponding to 50\%. 8 lesions $(22.22 \%)$ measured $<5 \mathrm{~cm}$ and 7 lesions $(19.44 \%)$ measured $10-15 \mathrm{~cm}$. The average size of the lesion is $7.986 \mathrm{~cm}$ in largest dimension. Of the 36 lesions studied, 25 lesions (69.44\%)are hyperechoic, 9 lesions (25\%) are mixed echogenic and 2 lesions (5.56\%) are predominantly hypoechoic.

Intralesional vascularity is seen in $83.33 \%$, as detected by color doppler. Intralesional necrosis is noted in $41.67 \%$ of the cases which occurs when the tumour outgrows its vascular supply. Karahan $\mathrm{OI}^{14}$ showed that the portal vein thrombosis was more frequently associated with lesions larger than $10 \mathrm{~cm}$, whereas in our study, portal vein thrombosis was seen in the tumour sizes ranging from $4.4 \mathrm{~cm}$ to $10 \mathrm{~cm}$. Peri-lesional halo could be demonstrated in 26 cases, corresponding to $72.22 \%$. Of all the lesions studied, no lesion showed intra lesional calcification, which is in contradiction to several studies, which found an incidence of intralesional calcifications, ranging from $3-25 \%{ }^{15,16}$. In this study, 35 lesions (97.22\%) showed enhancement in arterial phase and one lesion showed no enhancement $(2.78 \%)$. Venous phase washout was seen in 32 of the 35 lesions that showed enhancement in the arterial phase, corresponding to $91.43 \%$. While 3 lesions, corresponding to $8.57 \%$ did not show washout in the venous phase. These findings, are in close agreement with the findings made by K.H.Y. Lee $(2003)^{17}$, who have found that hypervascular component was seen in $96 \%$ of the cases, $86 \%$ of the lesions showed arterial phase enhancement and $76 \%$ of the lesions showed washout in the venous phase. Baron et $\mathrm{al}^{14}$ in their study on 66 patients found that $39 \%$ of the lesions are hyper attenuating in the arterial phase, another $39 \%$ are showing mixed attenuation and $21 \%$ are hypo attenuating. A well-defined delayed enhancing capsule could be demonstrated in 28 lesions (77.78\%). 34 lesions (94.44\%) showed T1 isointense signal, one $(2.77 \%)$ showed $\mathrm{T} 1$ Hyperintense signal and one $(2.77 \%)$ remained isointense. 35 lesions $(97.22 \%)$ showed T2 Hyperintense signal while one lesion $(2.77 \%)$ showed hypo intensity on T2.28 lesions (77.78\%) showed mosaic architecture while $8(22.22 \%)$ lesions did not. SGOT levels are raised in $100 \%$ of the patients while SGPT levels are raised in 83.33\%. Raised AFP values are noted in 35 cases (97.22\%), of which, 33 cases $(91.66 \%)$ showed a value $>1000 \mathrm{ng} / \mathrm{ml}$. Normal value could be observed in only one case.

\section{Conclusion}

HCC can present over a wide range of age groups and can have a wide spectrum of radiological appearances. Hence, no single feature can be pathognomonic of the diagnosis.

However, the most consistent findings in our study include:

USG: hyperechoic mass lesion with intralesional vascularity and a peripheral halo.

CECT: lesion showing enhancement in the arterial phase and washout in the venous phase with a well-defined capsule that enhances in the delayed phase and showing mosaic architecture.

MRI: T1 hypo intensity, T2 hyperintensity, with mosaic architecture. Biochemistry: Elevated AFP with deranged LFTs.

However, a tissue diagnosis is always desirable, whenever there is high index of suspicion, in lesions showing atypical pattern of imaging features.

\section{References}

1. Ferlay J, Soerjomataram I, Ervik M, et al. GLOBOCAN 2012 v1.0. Estimated cancer incidence, mortality, and prevalence worldwide. Available at http://globocan.iarc.fr. Accessed 28 March 2014.

2. World Health Organization Mortality Database. WHO Statistical Information System. 2008 Available at: http://www.who.int/whosis.

3. Colombo M, de Franchis R, Del Ninno E, et al. Hepatocellular carcinoma in Italian 
patients with cirrhosis. N Engl J Med. 1991;325:675-680. [PubMed]

4. Korean Liver Cancer Study Group and National Cancer Center, Korea. Practice guidelines for management of hepatocellular carcinoma 2009. Korean J Hepatol2009;15:391-423.

5. Cruite I, Tang A, Sirlin CB. ImagingBased Diagnostic Systems for Hepatocellular Carcinoma. American Journal of Roentgenology. 2013 Jul;201(1):41-55.

6. Teefey SA, Hildeboldt CC, Dehdashti F, Siegel BA, Peters MG, Heiken JP, et al. Detection of Primary Hepatic Malignancy in Liver Transplant Candidates: Prospective Comparison of CT, MR Imaging, US, and PET. Radiology. 2003 Feb 1;226(2):533-42.

7. Sangiovanni A, Manini MA, Iavarone M, Romeo R, Forzenigo LV, Fraquelli M, et al. The diagnostic and economic impact of contrast imaging techniques in the diagnosis of small hepatocellular carcinoma in cirrhosis. Gut. 2010;59:638644. [PubMed]

8. Peterson MS, Baron RL, Marsh JW Jr, Oliver JH 3rd, Confer SR, Hunt LE. Pretransplantation surveillance for possible hepatocellular carcinoma in patients with cirrhosis: epidemiology and CT- based tumor detection rate in 430 cases with surgical pathologic correlation. Radiology 2000;217:743- 749 .

9. Noguchi Y, Murakami T Kim T, Hori M, Osuga K, Kawata S, et al. Detection of hepatocellular carcinoma: comparison of dynamic MR imaging with dynamic double arterial phase helical CT. AJR Am J Roentgenol 2003;180:455-460.

10. D'Onofrio M, Faccioli N, Zamboni G et al (2008) Focal liver lesions in cirrohosis: value of contrast- enhanced ultrasonography compared with Doppler ultrasound and alpha-fetoprotein levels. Radiol Med 113:978-991

11. Baron RL, Oliver JH III, Confer S et al (1997) Screening cirrhosis for hepatocellular carcinoma (HCC) with helical contrast CT: specificity. Radiology 2005:143

12. Bruix J, Scherman M, Llovet JM et al (2001) Clinical managment of hepatocellular carcinoma. Conclusions of the barcellona-2000 EASL conference. Europe Assosation for the Study of the Liver. J Hepatol 35:421-430

13. Marin D, Di Martino M, Guerrisi A et al (2009) Hepatocellular carcinoma in patients with cirrhosis: qualitative comparison of gadobenatedimeglumine enhanced MR imaging and multiphasic 64section CT. Radiology 251:1-8

14. Karahan OI, Yikilnaz A, Lsin S, Orhan S. Characterization of Hepatocellular Carcinoma with Triphasic CT and Correlation with Histopathologic Findings. Acta Radiological 2003; 44(6):566-571

15. Itai V, Araki T, Furui S, Tasaka A. Differential diagnosis of hepatic masses on computed tomography, with particular reference to hepatocellular carcinoma. J Comput Assist Tomogr 1981;5:834-842

16. Teefey SA, Stephens OH, James EM, Charboneau JW, Sheedy PF II. Computed tomography and ultrasonography of hepatoma. ClinRadiol1986;37:339-345

17. K.H.Y. Lee, M.E. O' Malley, M.A. Haider, A. Hanbidge Triple-phase MDCT of HCC AJR 2004;182:643-649. 